\title{
A Short Duration Co-vibration Protocol of Antagonist Ankle Muscles to Induce Compensatory Postural Adjustments in Quiet Standing
}

\author{
Romain Tisserand $^{1,2}$, Yannick Fonollosa ${ }^{3}$, Thomas Robert ${ }^{1}$, Laurence Chèze ${ }^{1}$, Pascal Chabaud ${ }^{3, *}$ \\ ${ }^{1}$ Laboratoire de Biomécanique et Mécanique des Chocs IFSTTAR UMR_T9406, Université Claude Bernard Lyon 1, Villeurbanne, France \\ ${ }^{2}$ School of Kinesiology, University of British Columbia, Vancouver, British Columbia, Canada \\ ${ }^{3}$ Laboratoire Interuniversitaire de Biologie de la Motricité EA 7424, Université Claude Bernard Lyon 1, Villeurbanne, France
}

\section{Email address:}

romain.tisserand@ubc.ca (R. Tisserand), yannick.fonollosa@etu.univ-lyon1.fr (Y. Fonollosa), thomas.robert@ifsttar.fr (T. Robert), laurence.cheze@univ-lyon1.fr (L. Chèze), pascal.chabaud@univ-lyon1.fr (P. Chabaud)

${ }^{*}$ Corresponding author

\section{To cite this article:}

Romain Tisserand, Yannick Fonollosa, Thomas Robert, Laurence Chèze, Pascal Chabaud. A Short Duration Co-vibration Protocol of Antagonist Ankle Muscles to Induce Compensatory Postural Adjustments in Quiet Standing. American Journal of Psychiatry and Neuroscience. Vol. 5, No. 6, 2017, pp. 66-69. doi: 10.11648/j.ajpn.20170506.12

Received: October 5, 2017; Accepted: October 19, 2017; Published: November 16, 2017

\begin{abstract}
Vibration protocols classically used to alter kinesthetic information are limited for studying quick compensatory postural responses normally induced by external balance perturbations. To overcome these limitations, this study proposes a co-vibration protocol of ankle antagonist muscles: from a co-vibration baseline at $40 \mathrm{~Hz}$ a switch at $80 \mathrm{~Hz}$ is applied for 1 second but on the agonists only. This protocol induced both quick illusions and quick compensatory postural adjustments coherent with previous literature. This technical solution will be useful to build future protocols investigating the role of kinesthetic sensory information in quick compensatory postural responses.
\end{abstract}

Keywords: Postural Control, Compensatory Postural Adjustments (CPA), Kinesthesia, Co-vibration, Antagonist Muscles

\section{Introduction}

Following an external perturbation of standing posture stepping is a natural, common and efficient compensatory reaction to maintain balance and avoid falling $[1,2]$. It is known that kinesthetic sensory information plays a role in the triggering of compensatory reactions following a perturbation of standing posture, as the direction of postural instability affects information processing from ankle muscle receptors [3] and altered kinesthetic information reduces the amplitude of compensatory postural adjustments (CPA) [4]. However, the role of kinesthetic information in the triggering of protective steps remains largely unknown.

Tendinous vibration of ankle muscles is a classical method used to alter kinesthetic sensory information and study postural control in humans $[5,6]$. When applied during quiet stance, two phenomena are observed: an illusion of swaying in the opposite direction of the vibrated muscle followed by CPA moving the body in the direction of the vibrated muscle [5-12]. However, vibrators classically used to alter kinesthetic information need time to reach the desired frequency. As a consequence, previous studies used vibration periods of 2 seconds and longer (except for [5]) to trigger CPA when human subjects were standing quietly. In the context of a protective step, a quick postural reaction can easily be triggered with a perturbation shorter than a second [2]. Thus, the previous technical limitation reduces the feasibility of investigating the role of kinesthetic information. Moreover, studies usually described the characteristics of CPA without reporting the latency of the first oscillation of the center of pressure, which delimits the biomechanical postural response from the illusion integration period. However, this distinction is of interest in order to investigate whether a postural response is influenced by the central integration of the vibration or by the execution of the CPA, when tendinous vibrations are used.

To overcome the vibrators' limitations, a co-vibration protocol of antagonist muscles seems a relevant solution for several reasons [13]: (1) vibrating antagonistic muscles at the 
same frequency do not generate an illusion, (2) applying a co-vibration with different frequencies makes the illusion appear sooner than with a single muscle vibration, and (3) velocity of the illusion increases with the difference in vibration frequencies. The hypothesis of this study is that an initial co-vibration of antagonist ankle muscles at low frequency followed by a short vibration at high frequency on agonists muscles only would generate both illusion and CPA with short latencies. The two objectives of this study were to validate this protocol relative to previously published results and to determine the amplitudes and timings of CPA.

\section{Material \& Methods}

\subsection{Experiments}

Thirteen healthy adults ( 5 women, mean age 22.5 years old, height $1.72 \mathrm{~m}$ and weight $66.9 \mathrm{~kg}$ ) participated in this study approved by the local ethical committee (Lyon Sud-Est III). Subjects were asked to "maintain balance as naturally as possible" while standing quietly on two forceplates $(60 \mathrm{x} 40$ $\mathrm{cm}$, Bertec $\left.{ }^{\circledR}, \mathrm{OH}, \mathrm{USA}\right)$ sampled at $1000 \mathrm{~Hz}$. Subjects selfselected their feet position to be comfortable and were then equipped with 4 mechanical inertial vibrators (VB115, Vibrasens $\left(\right.$, Techno-Concept $\left({ }^{\circledR}\right)$. Vibrators were placed bilaterally on the gastrocnemius (GA) and tibialis anterior (TA) tendons and secured with elastic bands around subjects' legs. Each participant performed eight 15 -seconds trials with occluded vision: two "reference" trials (REF1 and REF2) and six "experimental" trials (EXP1 to EXP6). REF1 consisted in standing balance with no vibration. In REF2 a continuous bilateral co-vibration at $40 \mathrm{~Hz}$ was applied during the whole trial. Experimental trials consisted in a continuous bilateral co-vibration at $40 \mathrm{~Hz}$ with a bilateral change of the frequency to $80 \mathrm{~Hz}$ (starting randomly between 3 and 7 seconds after the beginning of the trial) for only one of the two muscle groups (GA or TA) and lasted for 1 second. Three EXP trials were performed per muscle group.

\subsection{Data Processing}

Trajectory of the center of pressure (CoP) was computed from the forceplate signals. Horizontal trajectory of the whole-body center of mass (CoM) was then computed using a lowpass filtering method [14]. Horizontal velocities of both the CoP and the CoM (VCoP and VCoM) were calculated from numerical differentiation of their respective trajectories, after applying a lowpass second-order Butterworth filter with a $20 \mathrm{~Hz}$ cutoff frequency. For REF1 and REF2 trials, the $95 \%$ ellipse area of the $\mathrm{CoP}\left(\mathrm{CoP}_{E}\right)$ was also measured [15]. In EXP trials, onset of $80 \mathrm{~Hz}$ vibration was identified as T0. Three timings $(\mathrm{T})$ were then defined relative to $\mathrm{T} 0$ from the anteroposterior component of the $\mathrm{CoP}$ displacement (Figure 1): the first instant of movement $\left(T C o P_{0}\right)$ and the first and second instants when it changed direction after $T C o P_{0}$ $\left(T C o P_{1}\right.$ and $\left.T C o P_{2}\right)$. These three instants were used to compute durations (D) and amplitudes (A) of the CoP movements: between $\mathrm{T} 0$ and $\operatorname{TCoP}_{0}\left(D C o P_{0}\right)$, between $\mathrm{TCoP}_{0}$ and $T \mathrm{CoP}_{1}\left(\mathrm{DCoP}_{1}\right.$ and $\left.A \mathrm{CoP}_{1}\right)$ and between $T C o P_{1}$ and $\mathrm{TCoP}_{2}\left(\mathrm{DCoP}_{2}\right.$ and $\left.A C o P_{2}\right)$. Velocity peak, both for the $\mathrm{CoP}$ and the $\mathrm{CoM}\left(V \operatorname{CoP}_{P}\right.$ and $\left.V \operatorname{CoM}_{P}\right)$ were also extracted.

For analysis, all GA trials were pooled together as well as all TA trials. Non-parametrical Wilcoxon tests were performed to compare the results between REF1 and REF2 and the results between TA and GA trials. Statistical tests were run with $\mathrm{R}$ software (version 3.3.1) and significance was identified with a two-tailed $\alpha$ level of 0.05 .

\section{Results}

The mean values of $C_{O} P_{E}\left(140\right.$ versus $\left.46 \mathrm{~mm}^{2}\right), V C o P$ (22,1 versus $\left.13,6 \mathrm{~mm} . \mathrm{s}^{-1}\right)$ and $\operatorname{VCoM}(5,51$ versus 3,40 $\left.\mathrm{mm} . \mathrm{s}^{-1}\right)$ were significantly increased in REF2 compared to REF1 (all $\mathrm{p}<0.001)$. However, no CPA were observed in REF2 (Figure 1, B).

In the GA trials, CoP oscillated first forward, moving the CoM backwards, and then moved backwards to slow the $\mathrm{CoM}$ and to bring it back to a quasi-static state of balance after one more oscillation (Figure 1, C). In TA trials, CoP moved in the opposite direction as in GA trials, with less oscillation (Figure 1, D).

$D C o P_{O}$ is inferior to $500 \mathrm{~ms}$ for both muscle groups on average, and even shorter for TA than for GA (Table 1). $D C o P_{1}$ was not different between muscle groups but $D C o P_{2}$ was significantly shorter in GA, for a similar amplitude. $A C o P_{1}$ was significantly greater in GA trials compared to TA trials while $\mathrm{ACoP}_{2}$ was not found different between muscle groups.

The differences observed in duration of the CPA concurs with the higher $V C_{C o P}$ and $V \operatorname{CoM}_{P}$ measured in GA compared to TA (Table 1). Peak velocities were observed after the end of the $80 \mathrm{~Hz}$ vibration (Figure 1, C \& D).

Table 1. Durations, amplitudes and velocity peaks of the CoP and CoM displacements induced by vibration (mean $+/-$ standard deviation). Results of the statistical non-parametric tests are provided on the right columns (W and p-value). NS indicates a $p>0.1$.

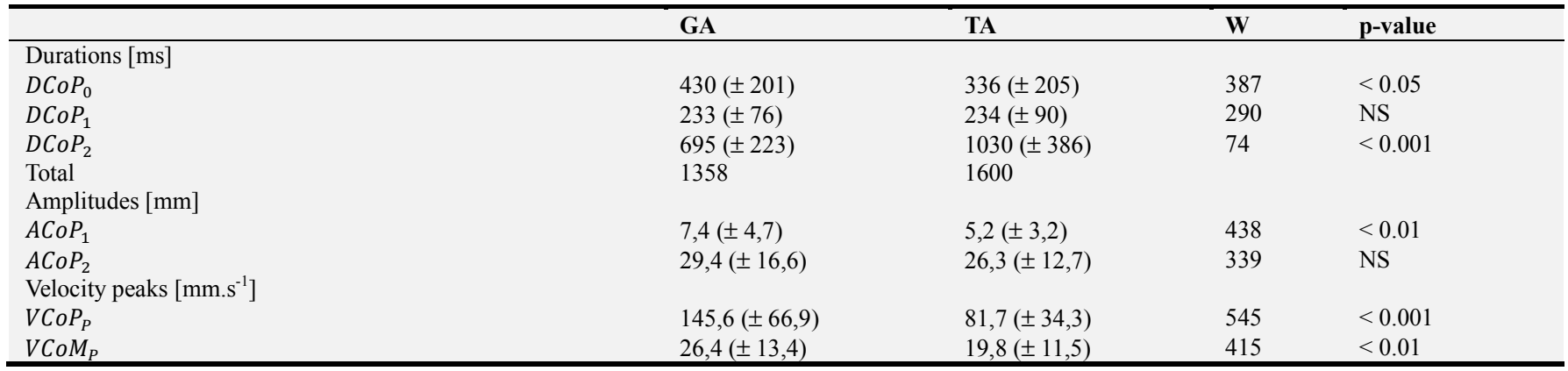




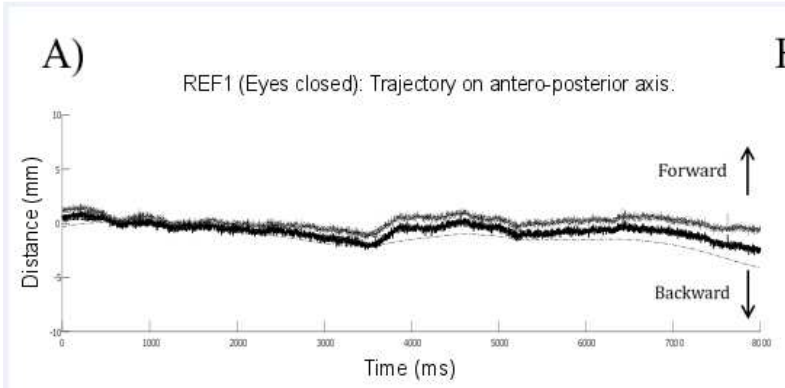

B) REF2 (Ey es closed + continuous $40 \mathrm{~Hz}$ vibration): Trajectory on antero-posterior axis

C)

D) Vibration at $80 \mathrm{~Hz}$ on GA: Trajectory and velocity on antero-posterior axis.
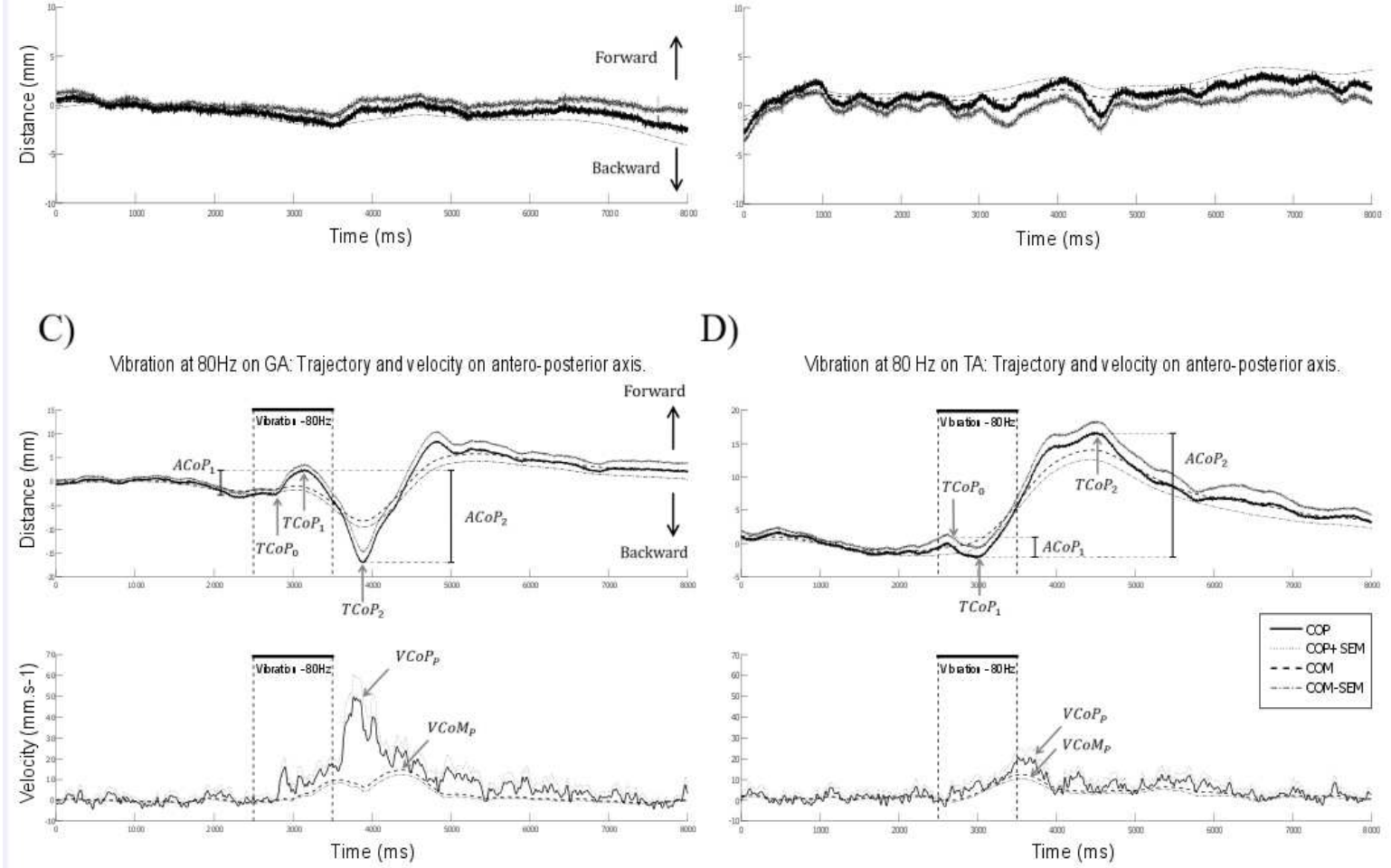

Figure 1. Averaged CoP and CoM trajectories and velocities during the four conditions on the anteroposterior axis. From top to bottom and from left to right: REF1 (panel A), REF2 (panel B), GA trials (panel C) and TA trials (panel D). For REF1 and REF2, only the CoP and CoM trajectories are presented. In panels $C$ and $D$, the trajectory is on the top and the velocity is on the bottom. In all panels, the CoP is presented with a solid black line and the CoM is presented with a dashed black line. CoP and CoM data are presented with plus or minus one standard error to the mean (SEM). Instants (TCoP $P_{0}$, TCoP $P_{1}$ and $\left.T C o P_{2}\right)$ and velocity peaks $\left(V C o P_{P}\right.$ and $\left.V C M_{P}\right)$ are reported with grey arrows and amplitudes $\left(A C o P_{1}\right.$ and $\left.A C o P_{2}\right)$ with double black braces. For clarity purpose, trajectories and velocities presented in all graphs have been aligned (fictive event in REF1 and REF2 trials and TO in GA and TA trials) and normalized on zero (baseline of $2500 \mathrm{~ms}$ preceding the event used for alignment). In the trajectory graphs, negative values indicate a backward direction while positive values indicate a forward direction.

\section{Discussion}

As in previous observations, co-vibrating the ankle antagonist muscles continuously at $40 \mathrm{~Hz}$ increased wholebody sway [11]. However, these postural corrections were not the CPA normally associated with single muscle tendon vibration. Thus, as already shown for arm muscles [13], both the illusion and the CPA are cancelled by the antagonists covibration when humans stand quietly.

The CoP oscillations consecutive to the short period of frequency change at $80 \mathrm{~Hz}$ were characteristics of previously reported postural responses to antagonist ankle muscles vibration: an anteroposterior displacement in the opposite direction of the vibrated muscle that drives the $\mathrm{CoM}$ in the direction of the vibrated muscle [5, 7, 9-12]. The first CoP oscillation occurred with a latency shorter than $500 \mathrm{~ms}$ in both muscle groups, which seems reasonably close to what was observed previously $[7,10-12]$ although not reported. Initial $\mathrm{CoP}$ movement amplitude $\left(A C o P_{1}\right)$ is found greater than in [10], indicating a greater sway to oppose the direction of the illusion. This suggests that the switch in frequency following a co-vibration period evoked the illusion sooner than with single muscle vibration [13]. The second oscillation of the CoP started about $235 \mathrm{~ms}\left(D C o P_{1}\right)$ after $T C o P_{0}$ in both muscle groups, for an average amplitude of 29 and $26 \mathrm{~mm}\left(\mathrm{ACoP}_{2}\right)$, respectively for GA and TA. These results were close to $[9,11]$ or much smaller $[7,12]$ than previous results. As participants reached maximal amplitude around $1500 \mathrm{~ms}$ after $\mathrm{T} 0$ in both muscle groups (see Total in Table 1), i.e. about $500 \mathrm{~ms}$ after the end of the $80 \mathrm{~Hz}$ vibration period, the differences measured in amplitude might be explained by the duration of the vibration used (from 2 up to 30 seconds in previous studies). Peak velocity always occurred after the end of the $80 \mathrm{~Hz}$ vibration period, indicating that the $\mathrm{CoP}$ catching back the CoM after its initial shift was the quickest adjustment performed during this protocol.

\section{Conclusion}

The main objective of this study was to induce illusion and CPA with short latencies by taking advantage of the covibration protocol and to reduce vibrators' latency. The proposed solution, switching the frequency from a $40 \mathrm{~Hz}$ covibration baseline to an $80 \mathrm{~Hz}$ vibration for 1 second, confirmed the hypothesis that this protocol will induce both quick vibration-evoked illusions and quick CPA in 
participants. Moreover, results provided in this study reveal the timing of two distinct periods: (1) the central integration of the kinesthetic information induced by tendinous vibration and (2) the execution of the CPA. These results will be used in future studies to determine when an external perturbation of quiet standing posture should be started, in order to investigate the role of kinesthetic sensory information in the triggering of quick compensatory reactions such as protective steps.

\section{Acknowledgements}

Authors thank Vincent Ballesio for technical support, Kean Kouakoua and Ludovic Miramand. Authors also thank Brandon Rasman for help in English redaction.

\section{Conflict of Interest Statement}

All the authors do not have any possible conflicts of interest.

\section{References}

[1] Maki BE, McIlroy WE. The role of limb movements in maintaining upright stance: the "change-in-support" strategy. Phys Ther 1997;77:488-507.

[2] Mille M-L, Rogers MW, Martinez K, Hedman LD, Johnson ME, Lord SR, et al. Thresholds for inducing protective stepping responses to external perturbations of human standing. J Neurophysiol 2003;90:666-74. doi:10.1152/jn.00974.2002.

[3] Ivanenko YP, Solopova IA, Levik YS. The direction of postural instability affects postural reactions to ankle muscle vibration in humans. Neurosci Lett 2000;292:103-6. doi:10.1016/S0304-3940(00)01438-5.

[4] Mohapatra S, Krishnan V, Aruin AS. Postural control in response to an external perturbation: Effect of altered proprioceptive information. Exp Brain Res 2012;217:197208. doi:10.1007/s00221-011-2986-3.
[5] Eklund G. General features of vibration-induced effects on balance. Ups J Med Sci 1972;77:112-24. doi:10.1517/03009734000000016.

[6] Roll JP, Vedel JP, Ribot E. Alteration of proprioceptive messages induced by tendon vibration in man: a microneurographic study. Exp Brain Res 1989;76:213-22. doi:10.1007/BF00253639.

[7] Hayashi R, Miyake A, Jijiwa H, Watanabe S. Postural readjustment to body sway induced by vibration in man. Exp Brain Res 1981;43:217-25. doi:10.1007/BF00237767.

[8] Roll R, Gilhodes JC, Roll JP, Popov K, Charade O, Gurfinkel $\mathrm{V}$. Proprioceptive information processing in weightlessness. Exp Brain Res 1998;122:393-402. doi:10.1007/s002210050527.

[9] Kavounoudias A, Gilhodes JC, Roll R, Roll JP. From balance regulation to body orientation: Two goals for muscle proprioceptive information processing? Exp Brain Res 1999;124:80-8. doi:10.1007/s002210050602.

[10] Smetanin BN, Popov KE, Kozhina G V. Postural reactions to vibratory stimulation of calf muscles under condition of visual inversion in human. Fiziol Cheloveka 2002;28:53-8.

[11] Thompson C, Bélanger M, Fung J. Effects of bilateral Achilles tendon vibration on postural orientation and balance during standing. Clin Neurophysiol 2007;118:2456-67. doi:10.1016/j.clinph.2007.08.013.

[12] Barbieri G, Gissot AS, Nougier V, Pérennou D. Achilles tendon vibration shifts the center of pressure backward in standing and forward in sitting in young subjects. Neurophysiol Clin 2013;43:237-42. doi:10.1016/j.neucli.2013.06.001.

[13] Gilhodes JC, Roll JP, Tardy-Gervet MF. Perceptual and motor effects of agonist-antagonist muscle vibration in man. Exp Brain Res 1986;61:395-402. doi:10.1007/BF00239528.

[14] Hof AL. Letter to the editor. J Biomech 2005;38:2134-5. doi:10.1016/S0021-9290(03)00251-3.

[15] Schubert P, Kirchner M. Ellipse area calculations and their applicability in posturography. Gait Posture 2014;39:518-22. doi:10.1016/j.gaitpost.2013.09.001. 